\title{
A New Traffic Congestion Detection and Quantification Method Based on Comprehensive Fuzzy Assessment in VANET
}

\author{
Lanlan Rui ${ }^{1}$, Yao Zhang ${ }^{1 *}$, Haoqiu Huang ${ }^{1}$, Xuesong Qiu ${ }^{1}$ \\ ${ }^{\mathbf{1}}$ State Key Laboratory of Networking and Switching Technology, Beijing University of Posts and \\ Telecommunications, Beijing 100876, China \\ [e-mail:\{llr, yaoayao, xsqiu\}@bupt.edu.cn] \\ *Corresponding author: Yao Zhang
}

Received May 11, 2017; revised July 19, 2017; accepted August 30, 2017;

published January 31, 2018

\begin{abstract}
Recently, road traffic congestion is becoming a serious urban phenomenon, leading to massive adverse impacts on the ecology and economy. Therefore, solving this problem has drawn public attention throughout the world. One new promising solution is to take full advantage of vehicular ad hoc networks (VANETs). In this study, we propose a new traffic congestion detection and quantification method based on vehicle clustering and fuzzy assessment in VANET environment. To enhance real-time performance, this method collects traffic information by vehicle clustering. The average speed, road density, and average stop delay are selected as the characteristic parameters for traffic state identification. We use a comprehensive fuzzy assessment based on the three indicators to determine the road congestion condition. Simulation results show that the proposed method can precisely reflect the road condition and is more accurate and stable compared to existing algorithms.
\end{abstract}

Keywords: VANET, vehicle clustering, comprehensive fuzzy assessment, traffic congestion detection, traffic state estimation 


\section{Introduction}

Recently, with the development of the automotive industry and the improvement of public living standards, the number of vehicles on the road is increasing rapidly. This causes serious traffic congestion in urban areas, which not only wastes the time of travelers, but also pollutes the environment. Thus, detecting traffic congestion is an urgent problem that needs to be solved worldwide. Majority of conventional detection methods are using fixed equipment, such as loop detectors [1, 2], microwave detectors [3], and sensors [4] to inspect vehicle speed, calculate mean speed, and then compare with the road threshold to estimate the road state and determine the level of road congestion. However, these methods have shortcomings such as high cost of maintenance and installation, limited range of detection, and difficulty in providing real-time traffic information.

With suitable enhancements, vehicular ad hoc networks (VANETs) have emerged as a promising and feasible way of detecting and controlling traffic congestion. VANETs consist of the communications between vehicles (V2V) and fixed roadside network infrastructure (V2R) [5], which are convenient to use and can provide accurate information. Several research proposals have been focused on detecting and reducing congestion using VANET. Jayapal and Roy [6] proposed a method for traffic congestion management, where congestion is confirmed by vehicle speed. Vehicles exchange and collect information via $\mathrm{V} 2 \mathrm{~V}$, determine the road state based on mean speed, and then each vehicle selects the road with highest average speed for the best route. In particular, velocity is considered as the only parameter for road condition. Lo and Kuo [7] also proposed a method of avoiding traffic congestion. A velocity map of the road where vehicles pass by is drawn by every vehicle, and is exchanged with neighbors in order to choose the road with low density. Similarly, the method proposed by Chi et al. [8] is for vehicles to detect the average speed and queue length, and then send this information to the roadside units (RSUs). The RSUs receive the information, compare with the standard threshold, and then estimate the road condition and adjust the traffic flow. Finally, the RSUs release the detection results to vehicles for them to choose the best path. Unfortunately, these methods cannot obtain real-time and accurate traffic information as vehicles are moving quickly, and there are limitations in the transmission range. In addition, majority of the existing proposals only consider a single factor, that is, velocity, and compare the average speed with a threshold to determine whether congestion has occurred, which have been verified to be less accurate and more erratic. Furthermore, there is no quantification of the road condition and traffic congestion.

To overcome some of the abovementioned shortcomings, we propose a new traffic congestion detection and quantification method in VANET. We know that vehicles move quickly, the network topology changes frequently in VANET, and moreover, every vehicle has its own transmission range. Therefore, we propose vehicle clustering to maintain a relatively stable environment to obtain real-time and accurate information. The RSU forms a 
cluster within its communication range and selects a cluster head $(\mathrm{CH})$ based on the priority of each cluster member (CM), where the priority is decided by mobility, distance to the RSU, and connectivity. The $\mathrm{CH}$ is responsible for receiving information from the $\mathrm{CMs}$ and then transmits such information to the traffic control center. As for identification of traffic congestion, because there is no clear boundary among road conditions, we use a comprehensive fuzzy assessment based on three factors, namely average speed, road density, and average stop delay to determine congestion levels by taking full consideration of the road condition. Simulation results show that the proposed method can precisely reflect the road condition and is more accurate and stable compared with existing algorithms. Our contribution is twofold:

1) We propose vehicle clustering for traffic information collection. This method ensures a relatively stable environment and improves real time and accuracy of collected information.

2) We classify traffic congestion into four levels based on transportation principles [9]. Three indicators of road condition are considered for the comprehensive fuzzy assessment; this significantly enhances the precision and stability of road condition identification.

The rest of the paper is organized as follows. In section II, we introduce vehicle clustering and $\mathrm{CH}$ selection algorithm for collecting information. The comprehensive fuzzy assessment algorithm is proposed in section III. Section IV describes the validation of the algorithm by simulation. Finally, we present the conclusion of this study in section V.

\section{Vehicle Clustering for Information Collection}

\section{A. Network Environment}

In this research, we use a link as the study object. A link is defined as a straight road section between two intersections [10]. The network environment consists of the RSU and the link. Every link has one lane, which means that vehicles are moving in the same direction, as shown in Fig. 1. We assume that every vehicle is equipped with GPS and can send/receive message from others. The message includes vehicle ID, speed, current position time, and a nonce. The format of the message packet is given in Table $\mathbf{1}$.

Table 1. Format of information message packet

\begin{tabular}{|l|l|l|l|l|}
\hline ID & Speed & Position & Time & Nonce \\
\hline
\end{tabular}




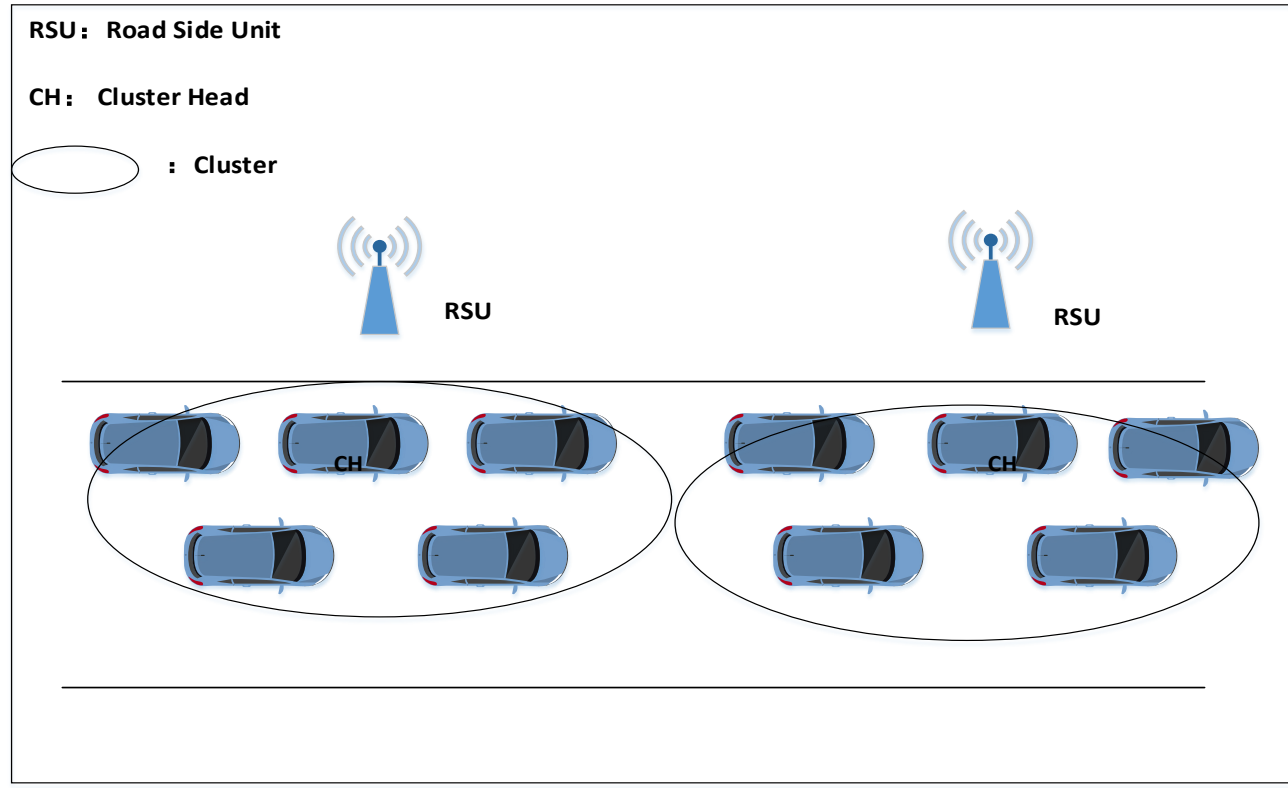

Fig. 1. Network environment

\section{B. Cluster Head Selection}

Based on [11, 12, 13], we construct our cluster algorithm and CH selection method.

Initially, the RSU aggregates the vehicles within its communication range and forms a cluster. Then, the RSU selects a $\mathrm{CH}$ in terms of mobility, distance, and connectivity. The weight value $\left(w_{C}\right)$, which is used to determine the possibility of each $\mathrm{CM}$ to be a $\mathrm{CH}$, is calculated as follows.

1) Mobility. We compare the current velocity $\left(V_{C}\right)$ and threshold velocity $\left(V_{T}\right)$ on the road. Vehicles with lower velocities $\left(V_{C} \leq V_{T}\right)$ are more likely to be chosen as $\mathrm{CH}$. That means that the lower the mobility, the higher the priority, as given by (1).

$$
w_{C 1} \propto \frac{1}{V_{C}}
$$

2) Distance. Consider the distance $\left(D_{R-i}\right)$ between the RSU and vehicles. The vehicle with the smallest distance has a higher priority and is more likely to act as $\mathrm{CH}$. This can be shown in (2) and (3).

$$
D_{R-i}=\sqrt{\left(x_{i}-x_{R}\right)^{2}+\left(y_{i}-y_{R}\right)^{2}}
$$

where $\left(x_{i}, y_{i}\right)$ is the vehicle position and $\left(x_{R}, y_{R}\right)$ is the position of the RSU.

$$
w_{C 2} \propto \frac{1}{D_{R-i}}
$$

3) Connectivity. We define the connectivity (C) of every vehicle based on its neighbor list (NL). The vehicle that has a longer neighbor list means that it has a higher degree of connection to other vehicles; hence, it has better chance to be a $\mathrm{CH}$. This can ensure that the $\mathrm{CH}$ have longer lifetime. The NL and C can be related by (4): 


$$
\mathrm{C} \propto \mathrm{NL}
$$

Because

$$
w_{C 3} \propto C
$$

we finally obtain

$$
w_{C 3} \propto N L
$$

Taking into account the three factors, according to above equations, the overall weight value $\left(w_{C}\right)$ for the chosen $\mathrm{CH}$ is calculated using (7).

$$
w_{C} \propto \frac{C}{V_{C} \cdot D_{R-i}}
$$

The vehicle that has the highest $w_{C}$ will be chosen as the $\mathrm{CH}$ of the cluster and the $\mathrm{CH}$ will be announced to other vehicles.

\section{Cluster Formation and Maintenance}

At the beginning, the RSU forms a cluster in terms of its communication range $\left(R_{i}\right)$, that is, vehicles with positions $\left(V_{p}\right)$ within $R_{i}$ are considered as CMs. Then, the RSU broadcasts a hello message to the CMs in order to announce the formation of the cluster. Vehicles receive the message and send a reply message to the RSU. Afterward, the RSU calculates and compares the $w_{C}$ of every vehicle and chooses the vehicle with the highest $w_{C}$ as the $\mathrm{CH}$. Finally, it puts the ID of the $\mathrm{CH}$ as cluster ID and announces it to the CMs.

As for cluster maintenance, it is achieved by the following steps.

1) The RSU checks the vehicles within its communication range periodically, where there are new vehicles joining and old vehicles leaving.

2) When a new vehicle enters into the communication range of the cluster, the $\mathrm{CH}$ and the new vehicle will compare their $w_{C}$. If $w_{C . C H} \geq w_{C . N e w}$, then the $\mathrm{CH}$ adds it into the CM list.

3) When any $\mathrm{CM}$ moves out of the communication range of the cluster, the $\mathrm{CH}$ deletes it from the CM list.

4) If the communication fails or the $\mathrm{CH}$ moves out, then one of the CMs that has the highest $w_{C}$ will be chosen as the new $\mathrm{CH}$.

Table 2. Clustering algorithm

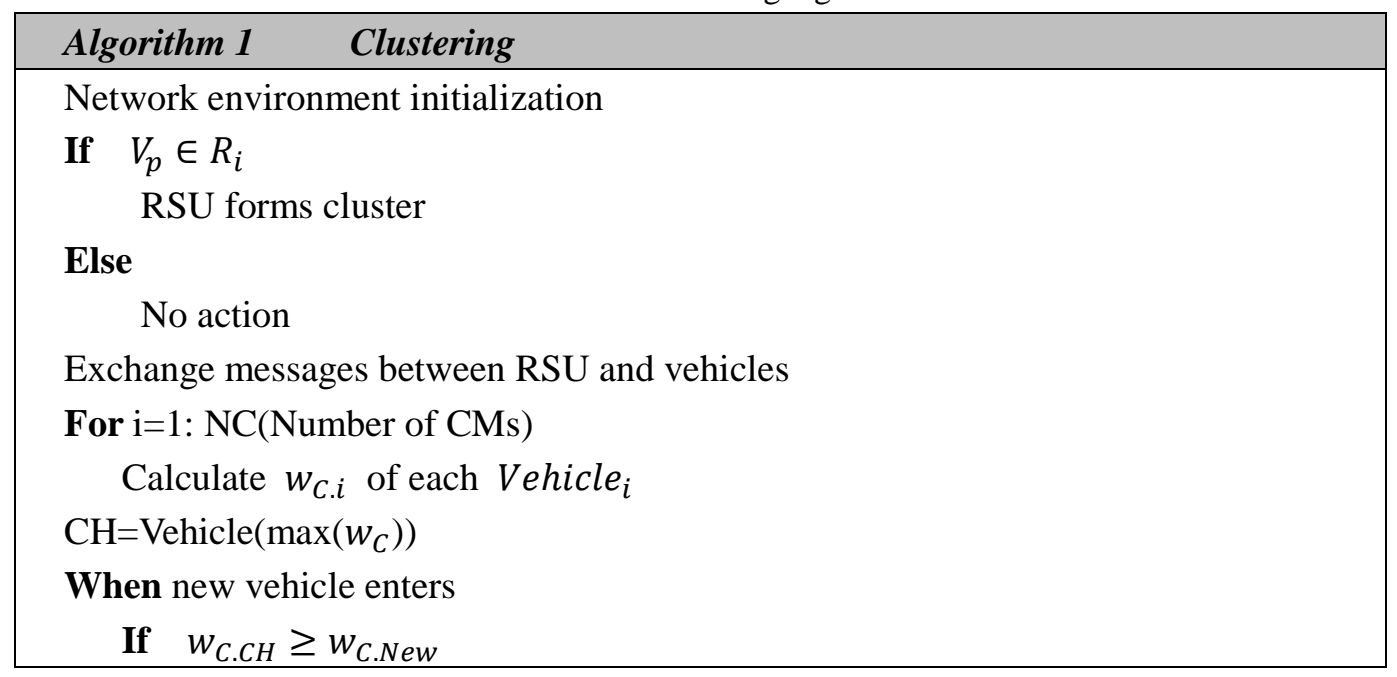




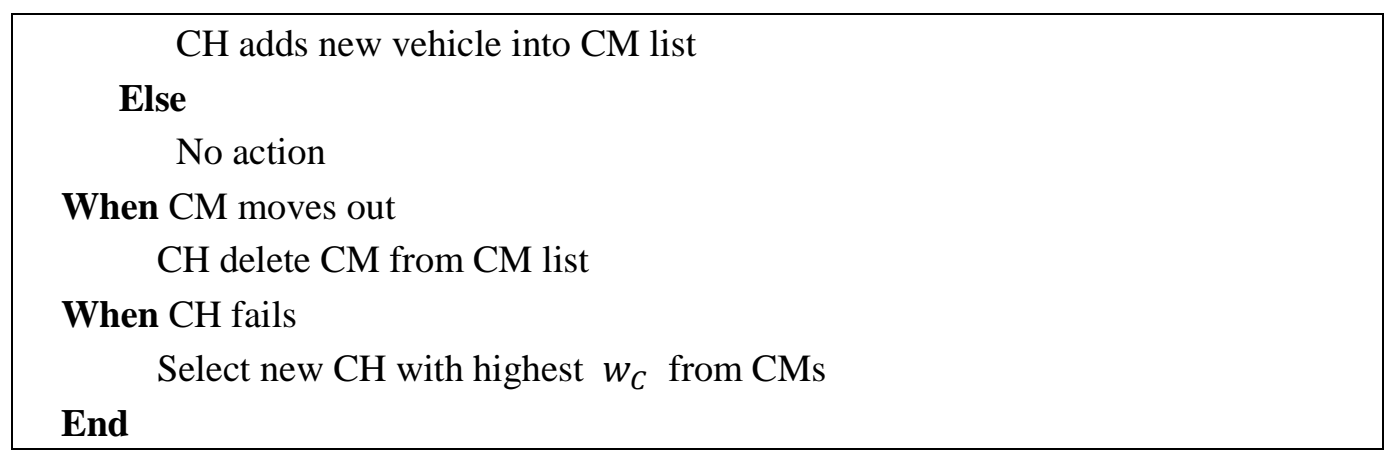

\section{Comprehensive Fuzzy Assessment}

After information collection, it is important to determine the level of traffic congestion using this information. In this part, we introduce a comprehensive fuzzy assessment [15]. As there is no clear boundary between traffic states, it is appropriate to use a comprehensive fuzzy assessment and its membership function to estimate road congestion [16, 17]. This method has a simple principle and requires a small amount of calculation. Furthermore, it can combine several indices better and obtain more accurate results. In addition, although road condition can be reflected by many evaluation indices, considering independence and practicability, we choose the average speed, road density, and average stop delay $[14,18]$ as the identification parameters.

\section{A. Overview of Comprehensive Fuzzy Assessment}

Defining $U, F$, and $R$ is the evaluation model of the comprehensive fuzzy assessment, where $\mathrm{U}=\left\{U_{1}, U_{2}, \ldots U_{m}\right\}$ is the set of evaluation factors, $U_{i}$ is the factor that affects the evaluation object, and $\mathrm{m}$ is the number of factors.

$\mathrm{F}=\left\{f_{1}, f_{2}, \ldots, f_{n}\right\}$ is the fuzzy assessment set, which is a set of possible evaluation results of the evaluation object, and $\mathrm{n}$ is the number of results.

$\mathrm{R}$ is the single factor evaluation set. For each $U_{i}(i=1,2, . . m)$, we select a membership function and calculate the membership $r_{i j}$ for $f_{j}(j=1,2, \ldots, n)$, which is the degree of membership for an evaluation object belonging to $f_{j}$ considering factor $U_{i}$. The closer $r_{i j}$ to 1 , the larger the degree of membership for the evaluation object belonging to $f_{j}$. Then, we obtain a single factor evaluation set $r_{i}=\left(r_{i 1}, r_{i 2}, \ldots r_{i j}\right)$ of $U_{i}$. The single factor evaluation set composed of $m$ factors is

$$
\begin{gathered}
\mathrm{R}=\left(r_{i j}\right)_{m \times n}=\left[\begin{array}{cccc}
r_{11} & r_{12} & \ldots & r_{1 n} \\
r_{21} & r_{22} & \ldots & r_{2 n} \\
\vdots & \vdots & & \vdots \\
r_{m 1} & r_{m 2} & \ldots & r_{m n}
\end{array}\right] \\
\left(i=1,2, . . m ; j=1,2, \ldots, n ; 0 \leq r_{i j} \leq 1\right)
\end{gathered}
$$

Finally, because the effect of the evaluation result on each factor is different, a weight set 
$\mathrm{W}=\left\{w_{1}, w_{2}, \ldots, w_{m}\right\}$ is necessary, where $w_{1}, w_{2}, \ldots, w_{m}$ are the weight values for factors $U_{1}, U_{2}, \ldots U_{m}$ respectively. Based on $\mathrm{W}$ and $\mathrm{R}$, we can obtain the comprehensive evaluation set $\mathrm{D}=\left\{d_{1}, d_{2}, \ldots, d_{n}\right\}$, as shown in (9), where $d_{j}$ is the membership of $f_{j}$ according to all the factors. We compare $d_{1}, d_{2}, \ldots, d_{n}$ and make a decision according to a maximum membership degree principle.

$$
\begin{gathered}
\mathrm{D}=W \bullet R=\left[\begin{array}{llll}
w_{1} & w_{2} & \cdots & w_{m}
\end{array}\right]\left[\begin{array}{cccc}
r_{11} & r_{12} & \cdots & r_{1 n} \\
r_{21} & r_{22} & \cdots & r_{2 n} \\
\vdots & \vdots & & \vdots \\
r_{m 1} & r_{m 2} & \cdots & r_{m n}
\end{array}\right]=\left[\begin{array}{llll}
d_{1} & d_{2} & \cdots & d_{n}
\end{array}\right] \\
\left(\sum_{i=1}^{m} w_{i}=1, i=1,2, \ldots, m\right)
\end{gathered}
$$

\section{B. Algorithm Implementation}

Combined with the actual road traffic condition and selected evaluation indicators, we achieve the comprehensive fuzzy assessment algorithm through the following steps. The notations used to describe the algorithm are listed in Table 3.

Table 3. Notations used for the algorithm

\begin{tabular}{|c|l|}
\hline Symbol & \multicolumn{1}{|c|}{ Meaning } \\
\hline $\boldsymbol{U}_{\mathbf{1}}=\boldsymbol{v}$ & Average speed \\
\hline $\boldsymbol{U}_{\mathbf{2}}=\boldsymbol{\rho}$ & Road density \\
\hline $\boldsymbol{U}_{\mathbf{3}}=\Delta \mathbf{t}$ & Average stop delay \\
\hline $\boldsymbol{f}_{\mathbf{1}}$ & Free-flow state of road \\
\hline $\boldsymbol{f}_{\mathbf{2}}$ & Slight congestion state of road \\
\hline $\boldsymbol{f}_{\mathbf{3}}$ & Moderate congestion state of road \\
\hline $\boldsymbol{f}_{\mathbf{4}}$ & Severe congestion state of road \\
\hline $\boldsymbol{r}_{\boldsymbol{i}}$ & $\begin{array}{l}\text { Degree of membership for road state belonging to } f_{j} \text { considering } U_{i} \\
(i=1,2,3 ; j=1,2,3,4)\end{array}$ \\
\hline $\boldsymbol{w}_{\boldsymbol{i}}^{\mathbf{1}}$ & $\begin{array}{l}\text { Weight of each factor calculated by analytic hierarchy process } \\
\text { (AHP) }(i=1,2,3)\end{array}$ \\
\hline $\boldsymbol{w}_{\boldsymbol{i}}^{\mathbf{2}}$ & Weight of each factor calculated by entropy method (ME) $(i=1,2,3)$ \\
\hline $\boldsymbol{w}_{\boldsymbol{i}}$ & Comprehensive weight of each factor $(i=1,2,3)$ \\
\hline $\boldsymbol{d}_{\boldsymbol{j}}$ & Membership of road state belonging to $f_{j}$ considering the three factors \\
\hline $\mathbf{M}$ & Number of evaluation factors \\
\hline $\mathbf{N}$ & Number of congestion levels \\
\hline
\end{tabular}

1) Establish evaluation factor set $U$

In this study, we choose three parameters and $U=\{v, \rho, \Delta t\}$. 


\section{2) Establish fuzzy assessment set $F$}

According to the urban traffic management evaluation system released by the Ministry of Public Security in China and the driver's acceptance of information, we divided road congestion degree into four levels, namely free-flow, slight, moderate, and severe [19]. Hence, we note that $\mathrm{F}=\left\{f_{1}, f_{2}, f_{3}, f_{4}\right\}$. The standard of indices is shown in Table 4 .

Table 4. Evaluation standard

\begin{tabular}{|lcccc|}
\hline Level & $\boldsymbol{f}_{\mathbf{1}}$ & $\boldsymbol{f}_{\mathbf{2}}$ & $\boldsymbol{f}_{\mathbf{3}}$ & $\boldsymbol{f}_{\mathbf{4}}$ \\
\hline$v(\mathrm{~km} / \mathrm{h})$ & $\geq 35$ & $25-30$ & $15-20$ & $\leq 10$ \\
$\rho(\mathrm{veh} / \mathrm{km} \cdot \mathrm{lane})$ & $\leq 25$ & $30-35$ & $40-45$ & $\geq 50$ \\
$\Delta \mathrm{t}(\mathrm{s})$ & $\leq 10$ & $20-45$ & $55-70$ & $\geq 80$ \\
\hline
\end{tabular}

\section{3) Select membership function}

Membership function selection is important because it directly affects the accuracy of judgment. According to the characteristics of the traffic flow and evaluation indices, we choose a trapezoidal function $[20,21]$ in this study.

i. Based on the evaluation standards in Table 4, we provide the membership function of $v$, which is shown in Fig. 2 and can be expressed as equation (10)-(13).

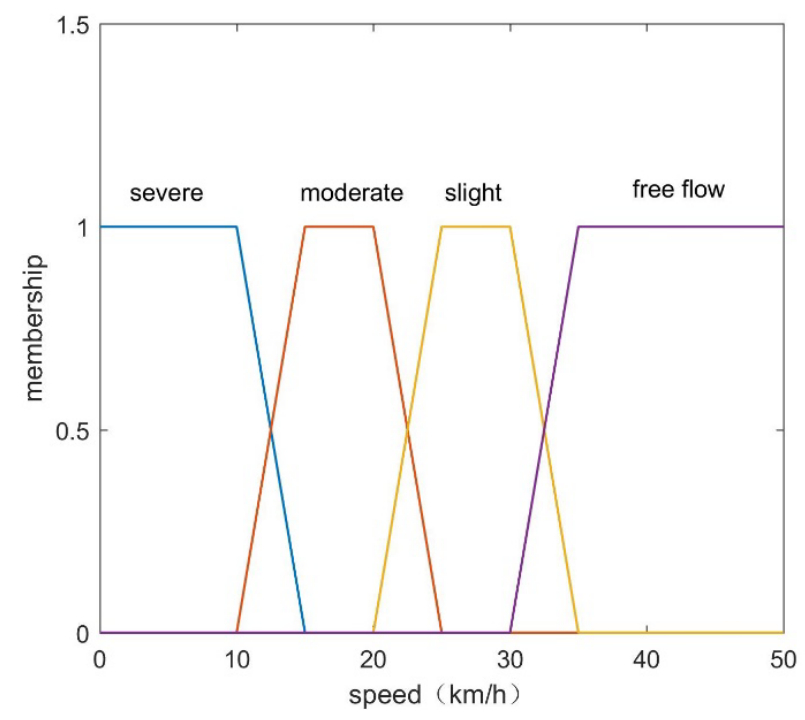

Fig. 2. Membership function of $v$

$$
r_{11}= \begin{cases}0, & v \leq 30 \\ \frac{v-30}{5}, & 30<v \leq 35 \\ 1, & v>35\end{cases}
$$$$
r_{12}= \begin{cases}0, & v \leq 20 \\ \frac{v-20}{5}, & 20<v \leq 25 \\ 1, & 25<v \leq 30 \\ \frac{35-v}{5}, & 30<v \leq 35 \\ 0, & v>35\end{cases}
$$ 


$$
r_{13}=\left\{\begin{array}{ll}
0, & 0 \leq v \leq 10 \\
\frac{v-10}{5}, & 10<v \leq 15 \\
1, & 15<v \leq 20 \\
\frac{20-v}{5}, & 20<v \leq 25 \\
0, & v>25
\end{array}(12) \quad r_{14}= \begin{cases}1, & v \leq 10 \\
\frac{15-v}{5}, & 10<v \leq 15 \\
0, & v>15\end{cases}\right.
$$

ii. According to the standards in Table 4, the membership function of $\rho$ is shown in Fig. 3 and is expressed as equation (14)-(17).

$$
\begin{array}{ll}
r_{21}=\left\{\begin{array}{ll}
1, \quad \rho \leq 25 \\
\frac{30-\rho}{5}, 25<\rho \leq 30 \\
0, \quad \rho>30
\end{array} \quad r_{22}= \begin{cases}0, & 0 \leq \rho \leq 25 \\
\frac{\rho-25}{5}, & 25<\rho \leq 30 \\
1, & 30<\rho \leq 35 \\
\frac{40-\rho}{5}, & 35<\rho \leq 40 \\
0, & \rho>40\end{cases} \right. \\
r_{23}=\left\{\begin{array}{ll}
0, \quad \rho \leq 35 \\
\frac{\rho-35}{5}, 35<\rho \leq 40 \\
1, \quad 40<\rho \leq 45 \\
\frac{50-\rho}{5}, 45<\rho \leq 50 \\
0, \quad \rho>50
\end{array} \quad r_{24}= \begin{cases}0, & \rho \leq 45 \\
\frac{\rho-45}{5}, & 45<\rho \leq 50 \\
1, & \rho>50\end{cases} \right.
\end{array}
$$

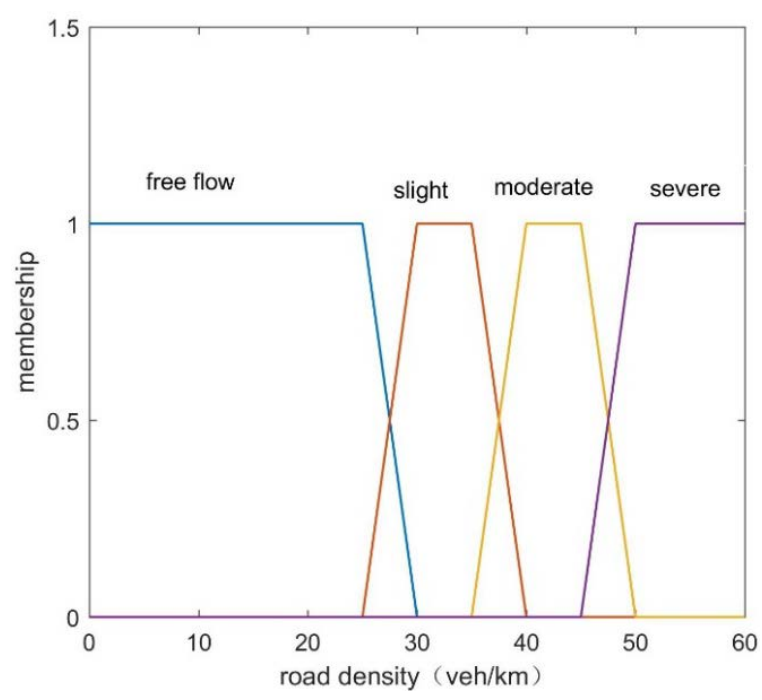

Fig. 3. Membership function of $\rho$

iii. Similarly, the membership function of $\Delta t$ can be expressed in Fig. 4 and equation (18)(21). 


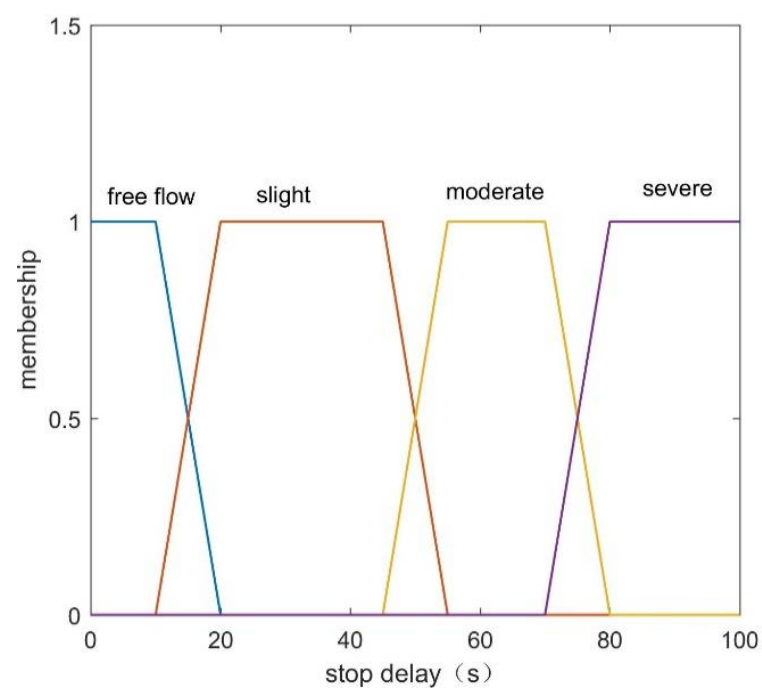

Fig. 4. Membership function of $\Delta t$

$r_{31}= \begin{cases}1, & \Delta t \leq 10 \\ \frac{20-\Delta t}{10}, & 10<\Delta t \leq 20 \\ 0, & \Delta t>20\end{cases}$

$r_{33}=\left\{\begin{array}{l}0, \quad \Delta t \leq 45 \\ \frac{\Delta t-45}{10}, 45<\Delta t \leq 55 \\ 1, \quad 55<\Delta t \leq 70 \\ \frac{80-\Delta t}{10}, \quad 70<\Delta t \leq 80 \\ 0, \quad \Delta t>80\end{array}\right.$

$$
\begin{gathered}
r_{32}= \begin{cases}0, & 0 \leq \Delta t \leq 10 \\
\frac{\Delta t-10}{10}, & 10<\Delta t \leq 20 \\
1, & 20<\Delta t \leq 45 \\
\frac{55-\Delta t}{10}, & 45<\Delta t \leq 55 \\
0, & \Delta t>55\end{cases} \\
r_{34}= \begin{cases}\frac{0 t-70}{\frac{\Delta t}{10}}, & \quad 70<\Delta t \leq 80 \\
1, & \Delta t>80\end{cases}
\end{gathered}
$$

\section{4) Confirm weight of factors}

The weight of factors can be determined using subjective and objective methods; however, using only a subjective or objective method is inadequate. A reasonable weight determination requires a combination of the two methods [21, 22]. Thus, in this study, we adopt the analytic hierarchy process (AHP) for the subjective method and the entropy method (ME) for the objective method. Finally, we calculate the comprehensive weight according to the two results.

\section{i. Analytic hierarchy process}

First, we analyze the importance of the evaluation factors based on expert surveys and establish a judgment matrix A: 


$$
\mathrm{A}=\left[\begin{array}{lll}
A_{11} & A_{12} & A_{13} \\
A_{21} & A_{22} & A_{23} \\
A_{31} & A_{32} & A_{33}
\end{array}\right]
$$

where $A_{i_{1} i_{2}}$ is the degree of importance of the factor $i_{1}$ relative to $i_{2}$, and its value is confirmed by the nine-quantile and expert surveys [21-23].

Then, we calculate the weight of each factor using equation (23):

$$
\omega_{\mathrm{i}}^{1}=\frac{\sqrt[m]{\prod_{i_{2}=1}^{m} A_{i_{1} i_{2}}}}{\sum_{i_{1}=1}^{m} \sqrt[m]{\prod_{i_{2}=1}^{m} A_{i_{1} i_{2}}}}(m=1,2,3 ; \quad \mathrm{i}=1,2,3,4)
$$

\section{ii. Entropy method}

Entropy is a measure of the uncertainty of information based on the probability theory, which indicates that the more divergent the data distribution, the greater its uncertainty [24, 25]. For each factor, if the values of its evaluation set $R_{i}$ is distributed divergently, the factor is definitely more important. In particular, if the values are equal, the index has no effect on determining the traffic states.

First, owing to the different dimensions of the evaluation indicators, normalization is required for a single factor evaluation set $\mathrm{R}=\left(r_{i j}\right)_{N \times M}$ in order to facilitate comparison. We use a method to normalize $\mathrm{R}$ to obtain $\mathrm{X}=\left(x_{i j}\right)_{N \times M}(\mathrm{~N}=4$ and $\mathrm{M}=3$ here). The equation is shown in (24).

$$
\begin{gathered}
x_{i j}=\frac{r_{i j}}{\sum_{\mathrm{j}=1}^{N} r_{i j}} \\
(i=1,2, \ldots, M ; j=1,2, \ldots, N)
\end{gathered}
$$

Then, we can calculate the entropy value of each factor using equation (25).

$$
\begin{gathered}
\mathrm{e}_{i}=-k \sum_{j=1}^{N} x_{i j} \ln x_{i j} \\
(i=1,2, \ldots, M ; j=1,2, \ldots, N ;)
\end{gathered}
$$

where $\mathrm{k}=1 / \mathrm{lnN}$

Finally, the weight is calculated according to the entropy, as shown in (26).

$$
\omega_{\mathrm{i}}^{2}=\left(1-e_{i}\right) / \sum_{i=1}^{M}\left(1-e_{i}\right)
$$

\section{iii. Comprehensive weight determination}


The comprehensive weight of evaluation factor is determined by a linear combination of $w_{i}^{1}$ and $w_{i}^{2}$.

$$
\omega_{\mathrm{i}}=\frac{\sqrt{\left(\omega_{i}^{1}\right)^{2}+\left(\omega_{i}^{2}\right)^{2}}}{\sum_{i=1}^{M} \sqrt{\left(\omega_{i}^{1}\right)^{2}+\left(\omega_{i}^{2}\right)^{2}}}, i=1,2, \ldots M
$$

\section{5) Make decision on the evaluation}

The final evaluation result is decided using the maximum membership degree principle. The comprehensive evaluation matrix $\mathrm{D}$ can be calculated using equation (9). If $d_{r}=$ $\max \left\{d_{j}, 1 \leq j \leq N\right\}$, then the congestion level belongs to level $\mathrm{r}$.

\section{Simulation Analysis}

\section{A. Experimental environment}

We use NS-3 for cluster formation simulation. We set nodes in NS-3 as mobile nodes to simulate the removable vehicles. The simulation area is a $2 \mathrm{~km} \times 1 \mathrm{~km}$ grid road network with many intersections, and the lane we consider is one direction. The nodes are deployed randomly on the topology. The setting of some specific parameters are listed in Table 5, and we can obtain the real simulation data through a tool named Trace Source in NS-3.

Moreover, the experimental data analyzed in our study are based on observations in Taoyuan Road, Beijing, which is a one-way street between two intersections. The road is 2 $\mathrm{km}$, and the data acquisition time is August 16, 2016, from 5:00 pm to 9:00 pm. We collect two sets of data at the same place and time: one is collected by an inductive loop detector and the other is from communications via vehicles. In addition, we specify the sampling interval as $1 \mathrm{~min}$, and the road state determination interval as $15 \mathrm{~min}$. We use Matlab to calculate and analyze the data and present the results.

Table 5. Simulation parameter settings

\begin{tabular}{|l|l|}
\hline \multicolumn{1}{|c|}{ Parameters } & \multicolumn{1}{c|}{ Value } \\
\hline Network topology & Sprint network topology \\
\hline Bandwidth & $1 \mathrm{Mbps}$ \\
\hline Communication radius & $50 \mathrm{~m}-200 \mathrm{~m}$ \\
\hline Area of the environment & $2 \mathrm{~km} \times 1 \mathrm{~km}$ \\
\hline Data link type & DLT_IEEE802_11_RADIO \\
\hline Packet size & 1040 bytes \\
\hline Motion model & IDM_LC \\
\hline Wireless propagation model & ConstantSpeedPropagationDelayModel \\
\hline Signal attenuation model & NakagamiPropagationLossModel \\
\hline MAC model & AdhocWifiMac \\
\hline Simulation time & $100 \mathrm{~s}$ \\
\hline
\end{tabular}




\section{B. Experimental results}

\section{1) Cluster formation}

We complete the cluster formation and $\mathrm{CH}$ selection using the method we mentioned above in our simulation environment, as shown in Fig. 5. The nodes in areas of different colors represent different clusters, and the remaining nodes are isolated nodes. Nodes in one cluster can communicate with each other and transmit messages to the $\mathrm{CH}$ in real time.

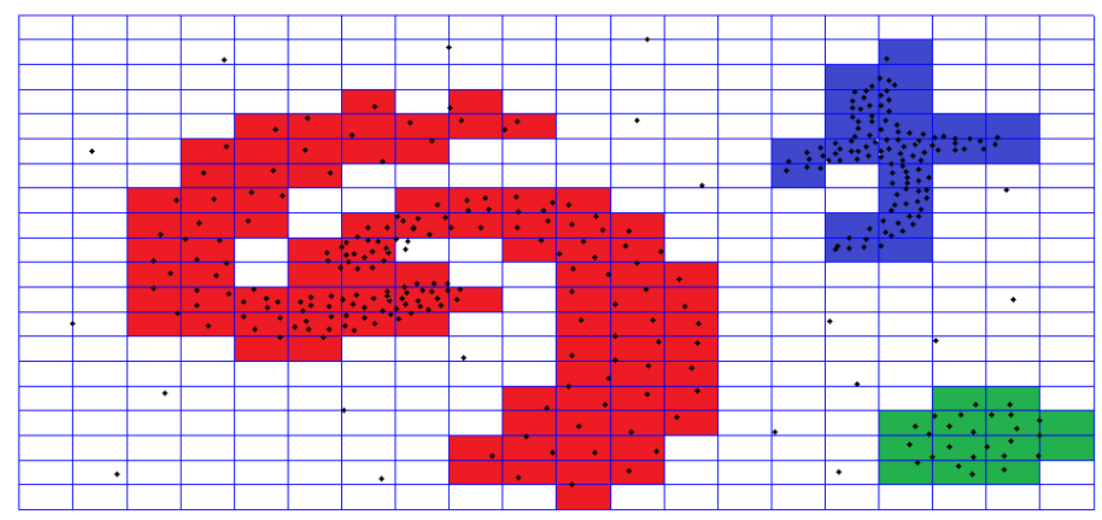

Fig. 5. Cluster formation

For further analysis, we also implement the basic cluster formation mentioned in reference [11] for comparison. We choose the times of $\mathrm{CH}$ change per second as evaluation index for cluster stability and the number of isolated nodes as evaluation index for the cluster coverage. The results are shown in Fig. 6 and Fig. 7.

As we can observe in Fig. 6, the times of $\mathrm{CH}$ change vary with the communication radius. We find that the fewer the times of $\mathrm{CH}$ change, the more stable the cluster structure. As shown in Fig 6, in our method, the $\mathrm{CH}$ is changed less frequently than the basic method, which means that the cluster format using our method is more stable. This is because when choosing the $\mathrm{CH}$, we consider the mobility, distance, and connectivity of a node comprehensively. Hence, the $\mathrm{CH}$ finally chosen is the one with higher stability.

As shown in Fig. 7, the number of isolated nodes in our proposed method is less than that of the basic method. As every node has useful information, we expect as many nodes as possible to be included in the cluster. The larger the coverage of the cluster, the more information we can obtain from more nodes. We can observe that there are less isolated nodes in our method, which means that our cluster has included more nodes and therefore, can collect more information. 


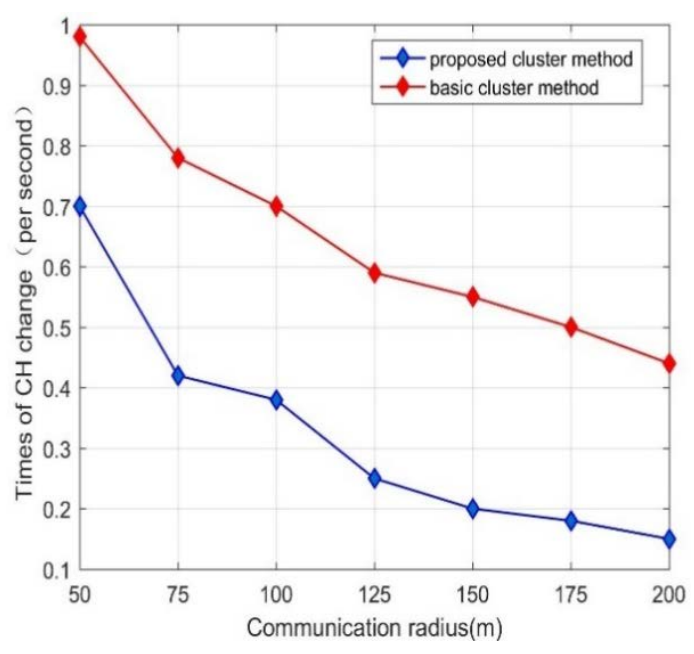

Fig. 6. Cluster stability analysis

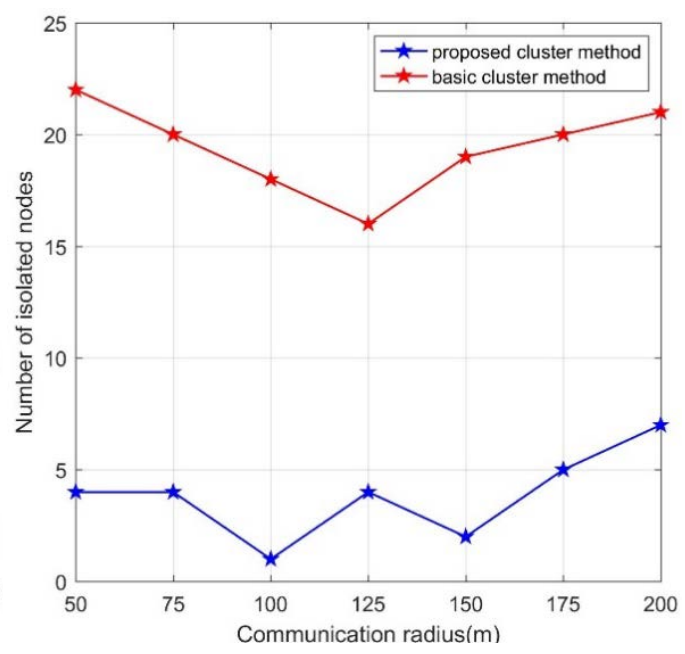

Fig. 7. Isolated nodes analysis

\section{2) Average speed}

We calculate the average speed every $15 \mathrm{~min}$ according to the data from different sources. The result is shown in Fig. 8. We can observe that although both data present the correct trend of the average speed, the result from vehicle communications achieves better fitting to the real average speed over the loop detector. For further comparison, we calculate the relative absolute error of the calculated values to the actual value, and draw the comparative graph, as shown in Fig. 9. We can observe that the average speed calculated based on the data from vehicle communications has a relatively smaller error. This is because vehicles can achieve real-time transmission of information, and after clustering, each $\mathrm{CH}$ will be responsible for the specific transmission range, and there is no transmission blind area. On the contrary, the information collected by the loop detector has a certain delay, and owing to the scope of transmission limitations, there will be undetected vehicles. Thus, the information collected via vehicle communications is more accurate compared with that of fixed equipment. 


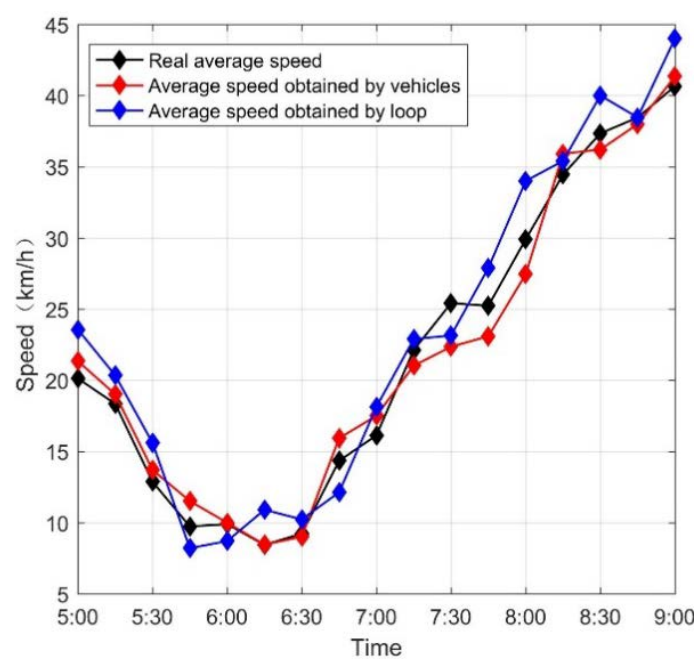

Fig. 8. Comparison of speed

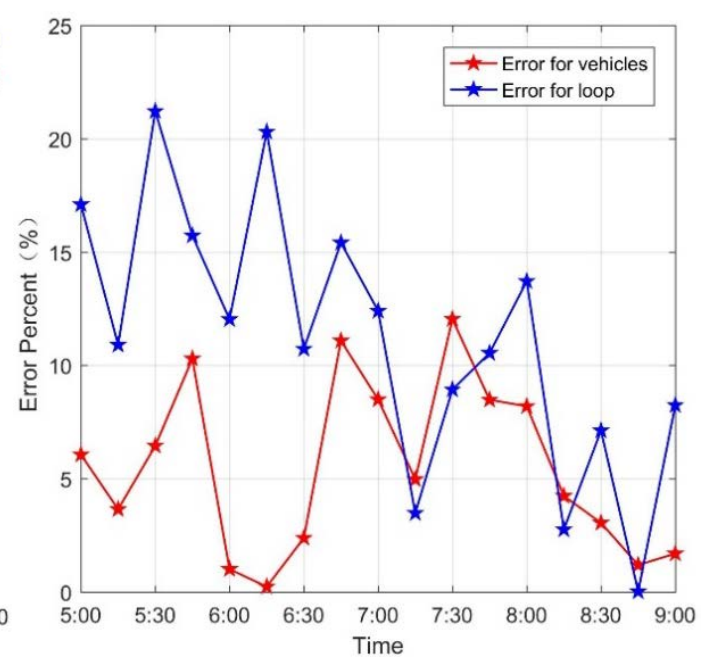

Fig. 9. Comparison of absolute error

\section{3) Determination results}

We set the state discrimination interval to $15 \mathrm{~min}$; thus, the observation time is divided into 16 discrimination periods. We process the data collected by vehicle communications, and calculate the values of the three indicators every $15 \mathrm{~min}$, as shown in Table 6 and Fig. 10. We can observe that during 5:00 pm-7:00 pm, owing to the peak of getting off from work, the average speed is very slow, and the road density and average stop delay are relatively high. After 7:00 pm, the road condition improves: the average speed increases and the road density and average stop delay are gradually becoming smaller.

Table 6. Values of indices

\begin{tabular}{|c|c|c|c|c|c|c|c|}
\hline Time & $v(\mathbf{k m} / \mathbf{h})$ & $\rho($ veh/km·lane) & $\Delta t(s)$ & Time & $v(\mathrm{~km} / \mathrm{h})$ & $\rho$ (veh/km·lane) & $\Delta t(s)$ \\
\hline $5: 00-5: 15$ & 19.01 & 25 & 22.34 & $7: 00-7: 15$ & 21.02 & 37 & 59.21 \\
\hline $5: 15-5: 30$ & 13.70 & 36 & 25.23 & $7: 15-7: 30$ & 22.35 & 37 & 58.48 \\
\hline $5: 30-5: 45$ & 11.51 & 51 & 76.56 & $7: 30-7: 45$ & 23.09 & 38 & 52.79 \\
\hline $5: 45-6: 00$ & 9.99 & 46 & 81.23 & $7: 45-8: 00$ & 27.45 & 35 & 46.89 \\
\hline $6: 00-6: 15$ & 8.45 & 38 & 80.34 & 8:00-8:15 & 35.91 & 33 & 44.60 \\
\hline $6: 15-6: 30$ & 9.01 & 39 & 81.34 & $8: 15-8: 30$ & 36.20 & 32 & 38.78 \\
\hline $6: 30-6: 45$ & 15.93 & 39 & 59.01 & 8:30-8:45 & 37.99 & 25 & 30.23 \\
\hline $6: 45-7: 00$ & 17.50 & 49 & 65.23 & 8:45-9:00 & 41.34 & 21 & 23.12 \\
\hline
\end{tabular}




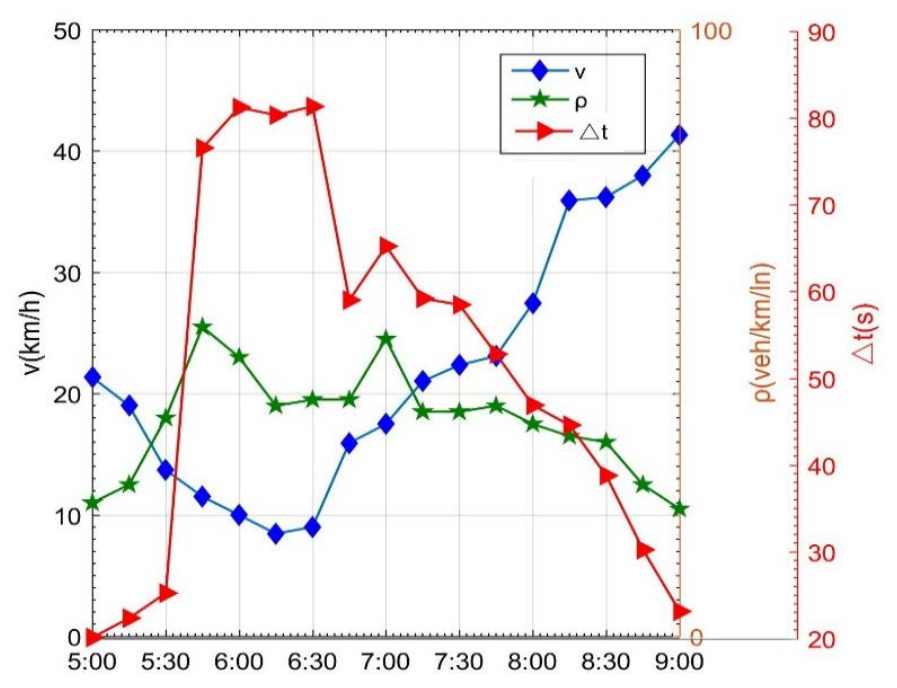

Fig. 10. Values of indices

We calculate the traffic condition result for each discrimination period using different methods, and the results are shown in Figs. 11-14. Figs. 11-13 show the results calculated using a single factor, which are obtained by comparing the average values with a presupposed threshold. Fig. 14 shows the calculated result using the comprehensive fuzzy method based on the three factors. In the figures, 1 represents the free-flow state, 2 the slight state, 3 the moderate state, and 4 the severe state.

By comparing Figs. 11-13 with Fig. 14, we can observe that the results using a single factor have larger fluctuations and lesser accuracy. However, by using the comprehensive fuzzy assessment, the judgment result has better stability and is also consistent with the actual traffic condition of the road because the traffic state always maintains a relative stability in a period of time instead of fluctuating. Moreover, 5:00 pm to 7:00 pm is the peak period; thus, there is severe traffic congestion on the road. After 7:00 pm, the number of vehicles decreases, the traffic congestion eases, and the road becomes gradually smooth. The result reflects the road condition accurately. In conclusion, the proposed algorithm has an advantage over other methods in terms of accuracy and stability.

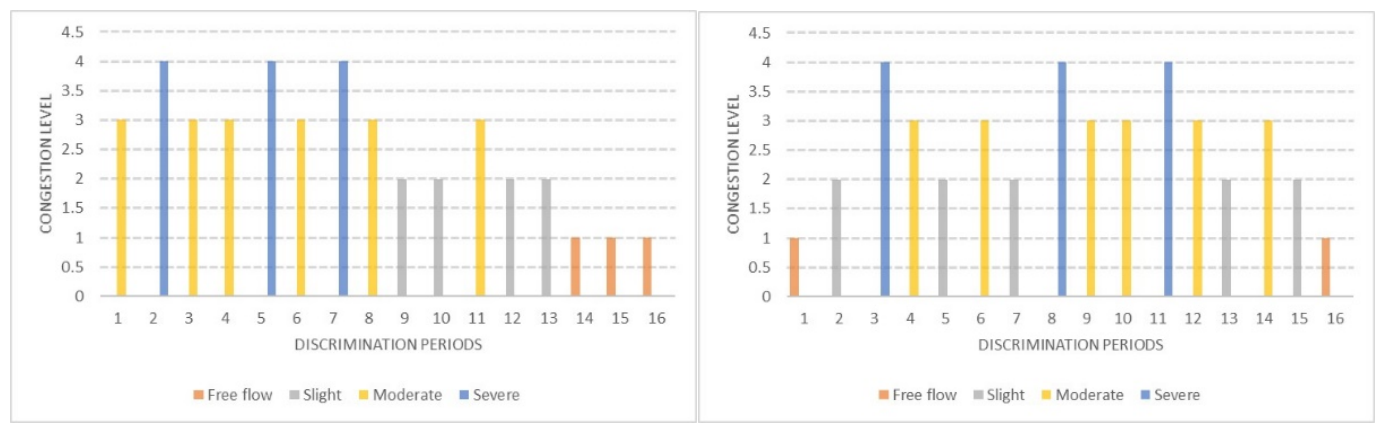

Fig. 11. Result based on average speed

Fig. 12. Result based on road density 


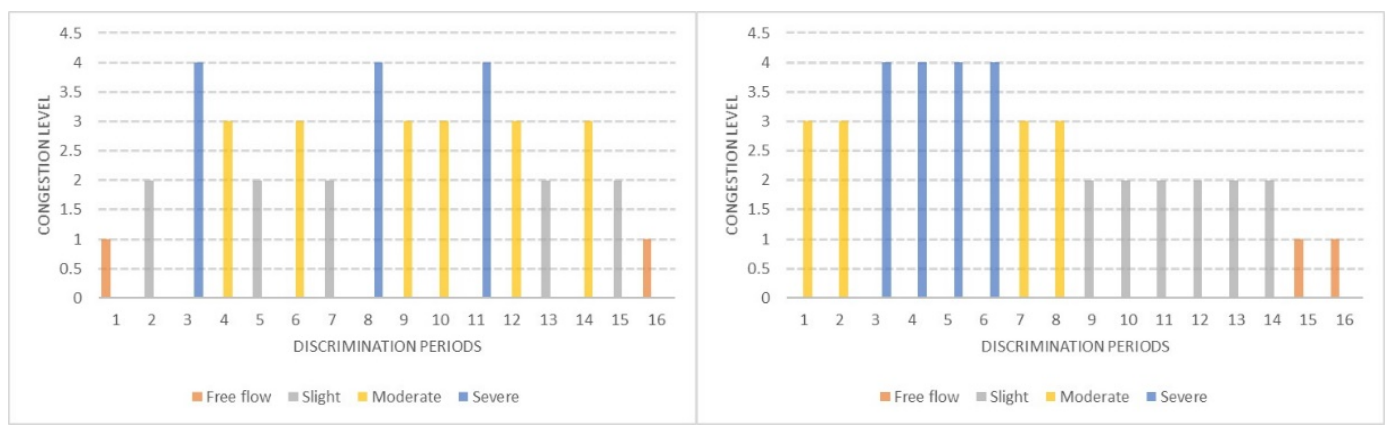

Fig. 13. Result based on average stop delay $\quad$ Fig. 14. Result using comprehensive fuzzy assessment

On the other hand, Fig. 15 shows the congestion estimation at different locations and time in two and three dimensions respectively. The figure allows an appreciation on how the traffic congestion evolves in space and time. In the figure, 1 is free-flow state and 4 is severe congestion. As observed during the peak hours, the middle of the road is the most congested area but the congestion gradually declines from the middle to both sides. In general, the congestion in the middle is more severe than that in both edges of the road. This is consistent with the real situation: as the detected road is a segment between two intersections, the road edge is near the intersection where the vehicular flow is much faster; thus, congestion easily reduces at the edge. On the contrary, the middle of the road is more prone to traffic congestion owing to the slow vehicular movement. Overall, the figure reflects a precise congestion situation in space and time.
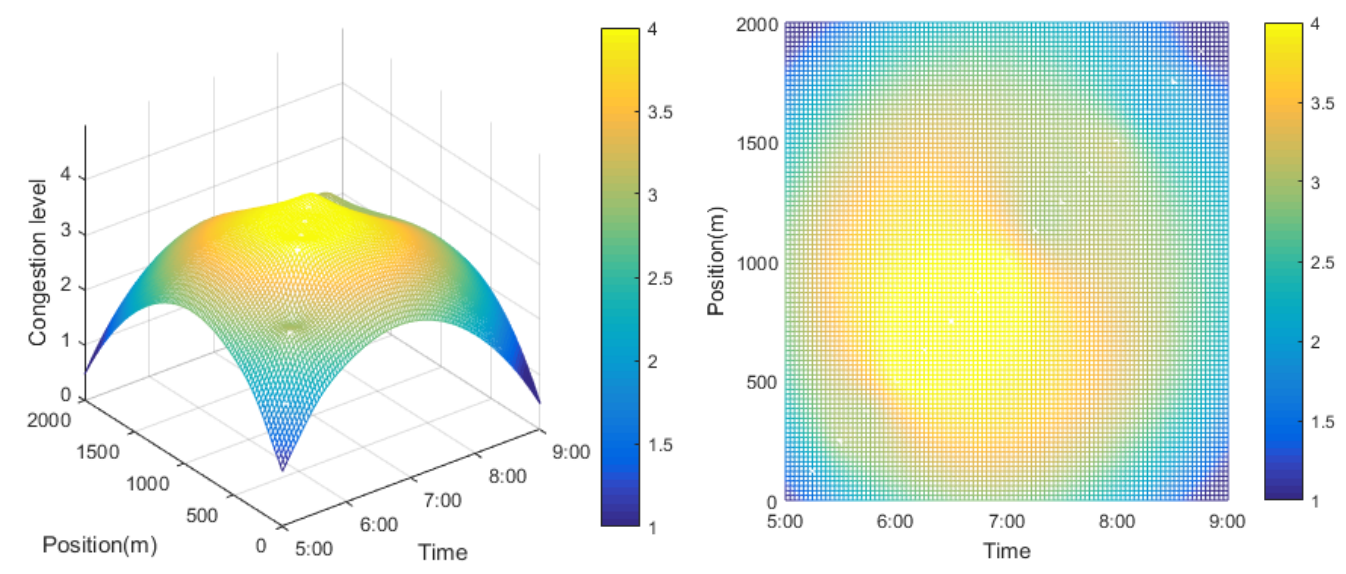

Fig. 15. Congestion in space and time

\section{Conclusion}

With the gradual increase of traffic congestion, strategies to address the problem have become a major public concern. In this study, we propose a traffic congestion detection and quantification method in VANET. We use vehicle clustering to collect information, where the 
$\mathrm{CH}$ is responsible for collecting information from the CMs and sending it to the traffic control center. In addition, we quantify traffic congestion by dividing it into four levels based on related principles. Finally, a comprehensive fuzzy assessment is adopted to identify the level of traffic congestion taking into account the average speed, road density, and average stop delay. Simulation results show that the method we proposed is capable of precisely reflecting the road condition and is more accurate and stable compared with existing algorithms.

\section{ACKNOWLEDGMENT}

The work presented in this paper was supported by the National Natural Science Foundation of China (61302078, 61372108), 863 Program (2011AA01A102), and Beijing Higher Education Young Elite Teacher Project (YETP0476).

\section{References}

[1] Matthew Wright, Roberto Horowitz, "Fusing loop and GPS probe measurements to estimate freeway density," IEEE Transactions on Intelligent Transportation Systems, vol.17, no.12, pp. 3577-3590, 2016. Article (CrossRef Link)

[2] David Guibert, Sio-Song leng, et al., "Robust blind deconvolution process for vehicle reidentification by an inductive loop detector," IEEE Sensors Journal, vol.14, no.12, pp. 4315-4322, 2014. Article (CrossRef Link)

[3] Mandalk, Sen A, et al., "Road traffic congestion monitoring and measurement using active RFID and GSM technology," in Proc. of International IEEE Conference on Intelligent Transportation Systems, pp. 1375-1379, 2011. Article (CrossRef Link)

[4] Qifan Wei, Bo Yang, "Adaptable vehicle detection and speed estimation for changeable urban traffic with anisotropic magnetoresistive sensors,” IEEE Sensors Journal, vol.17, no.7, pp. 2021-2028, 2017. Article (CrossRef Link)

[5] Marco Gramaglia, Maria Calderon, “ABEONA Monitored Traffic: VANET-Assisted Cooperative Traffic Congestion Forecasting,” IEEE Vehicular Technology Magazine, vol.9, no.2, pp. 50-57, 2014. Article (CrossRef Link)

[6] Cynthia Jayapal, S.Sujith Roy, "Road traffic congestion management using VANET," in Proc. of International Conference on Advances in Human Machine Interaction (HMI), pp. 1-7, 2016. Article (CrossRef Link)

[7] Chun-Chih Lo, Yau-Hwang Kuo, "Traffic-aware routing protocol with cooperative coverage-oriented information collection method for VANET," IET Communications, vol. 11, no.3, pp. 444-450, 2017. Article (CrossRef Link)

[8] Jeonghee Chi, Sunyoung Do,Soyoung Park, "Traffic flow-based roadside unit allocation strategy for VANET,” in Proc. of International Conference on Big Data and Smart Computing, pp. 245-250, 2016. Article (CrossRef Link)

[9] Yang Zhaosheng, Zhang Maolei, "A model for road state analysis based on fuzzy comprehensive evaluation,” Journal of Highway and Transportation Research and Development, vol. 27, no.9, pp. 121-126, 2010. Article (CrossRef Link)

[10] Ning Zhang, Jianmin $\mathrm{Xu}$, “An approach for real-time urban traffic state estimation by fusing multisource traffic data," Proceedings of the 10th World Congress on Intelligent Control and Automation, pp. 4077-4081, 2012. Article (CrossRef Link) 
[11] A.V. Sutagundar, Pampapati Hubballi, Ravikant Belagali, “Stablility Oriented Cluster Dynamism in VANET," in Proc. of International Conference on Computation System and Information Technology for Sustainable Solutions, pp. 117-120, 2016.

Article (CrossRef Link)

[12] Huixian Wang, Ren Ping Liu, "VANET modeling and clustering design under practical traffic, channel and mobility conditions,” IEEE Transactions on Communications, vol. 63, no. 3, pp. 870-881, 2015. Article (CrossRef Link)

[13] Pampapati Hubballi, A. V. Sutagundar, Ravikant Belagali, "Agent based dynamic clustering for hybrid VANET (ADCHV)," in Proc. of IEEE International Conference on Recent Trends in Electronics, Information \& Communication Technology, pp. 382-386, 2016.

Article (CrossRef Link)

[14] Nikolaos Bekiaris-Liberis, Claudio Roncoli, "Highway traffic state estimation with mixed connected and conventional vehicles,” IEEE Transactions on Intelligent Transportation Systems, vol.17, no.12, pp. 484-3497, 2016. Article (CrossRef Link)

[15] Tian S Y, Liu W M, "Research on the road section of the real time traffic conditions base on fuzzy comprehensive evaluation,” Science Technology and Engineering, vol. 10, no. 29, pp.7206-7209, 2010. Article (CrossRef Link)

[16] K Chen , Z Li, "Prediction of Traffic State Based on Fuzzy Logic in VANET," Information Technology Journal , vo.12, no. 18, pp.4642-4646, 2013.

Article (CrossRef Link)

[17] L Nassar, F Karray, "Fuzzy Logic in VANET context aware Congested Road and Automatic Crash Notification,” in Proc. of IEEE International Conference on Fuzzy Systems , pp. 1031-1037, 2016. Article (CrossRef Link)

[18] Minal Bhandarkar, Tejashri Waykole, "Vehicular mechanical condition determination and on road traffic density estimation using audio signals," in Proc. of International Conference on Computational Intelligence and Communication Networks, pp. 395-401, 2014.

Article (CrossRef Link)

[19] Ministry of Construction, China. Evaluation Index System for Urban Traffic Management [R]. 2012. Article (CrossRef Link)

[20] Christian A. Rojas, Jose R. Rodriguez, "Multiobjective fuzzy-decision-making predictive torque control for an induction motor drive,” IEEE Transactions on Power Electronics, vol.23, no.8, pp. 6245-6260, 2017. Article (CrossRef Link)

[21] Zhiquan Lai, Yongjun Shen, "A security risk assessment method of website based on threat analysis combined with AHP and entropy weight," in Proc. of 2016 7th IEEE International Conference on Software Engineering and Service Science (ICSESS), pp. 481-484, 2016.

Article (CrossRef Link)

[22] Ana Riza F. Quiros, Rhen Anjerome Bedruz, "Machine vision of traffic state estimation using fuzzy logic,” in Proc. of 2016 IEEE Region 10 Conference (TENCON), pp. 2104-2109, 2016. Article (CrossRef Link)

[23] Guangyu Zhu, Jianjun Chen, "Fuzzy c-means clustering identification method of urban road traffic state," in Proc. of $201512^{\text {th }}$ International Conference on Fuzzy Systems and Knowledge Discovery (FSKD), pp. 302-307, 2015. Article (CrossRef Link)

[24] Pan Y Z, "Study on evaluation of logistics finance regulatory service providers based on fuzzy multi-attribute decision making,” Logistic Technology, vol.32, no.7, pp. 245-248, 2013.

Article (CrossRef Link)

[25] Wu G X, Peng H J, “A multi-attribute decision making model for supply chain partner selection in manufacturing enterprises,” Industrial Engineering Journal, vol.13, no.2, pp.91-93, 2010. Article (CrossRef Link) 


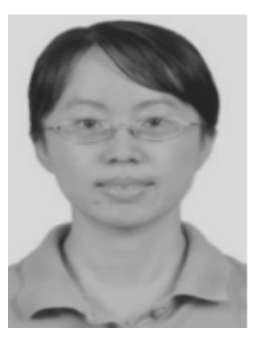

Lanlan Rui: female, Associate professor, master tutor in Beijing University of Posts and Telecommunications, research direction for communication software and network management.

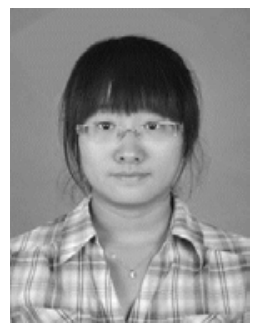

Yao Zhang: Master in Beijing University of Posts and Telecommunication, main research direction for network management.

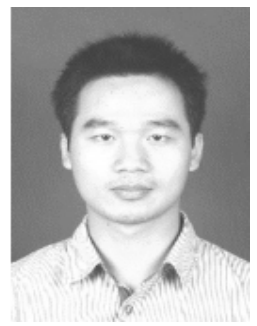

Haoqiu Huang: Doctor in Beijing University of Posts and Telecommunication, main research direction for network management.

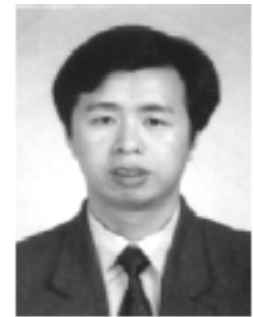

Xuesong Qiu: male, Professor and doctoral tutor in Beijing University of Posts and Telecommunications, research direction for network management and communication software. 